\title{
Physician Perception and Patient Input Are Important Influences in the Treatment of Hypothyroidism
}

\section{Charles H. Emerson}

Division of Endocrinology, Department of Medicine, University of Massachusetts School of Medicine, Worcester, Massachusetts, U.S.A.

\section{Review of Two Articles:}

1. Bellastella G, Caputo M, Maiorino MI, Longo M, Scappaticcio L, Giugliano D, Esposito K 2019 EMPATHY: a new tool for identifying the most suitable thyroxine formulation in hypothyroid patients. Thyroid 29:928-933. PMID: 30963820.

2. Esfandiari NH, Reyes-Gastelum D, Hawley ST, Haymart MR, Papaleontiou M 2019 Patient requests for tests and treatments impact physician management of hypothyroidism. Thyroid. Epub 2019 Oct 10. PMID: 31436135.

\section{SUMMARY}

\section{Background}

It has been recognized for many years that therapeutic decisions in clinical practice are not one-way channels, conveying simply directions and decisions from health care providers to their patients. Rather, in arriving at treatment choices, information and opinions are exchanged between the two parties. With the exception of a fairly robust literature in primary care journals $(1,2)$, however, there seems to be less attention paid to two-way patient-physician interactions in the specialties, including endocrinology. This may be changing, as demonstrated by the content of more recent American Thyroid Association meetings and the two publications reviewed here. This first of these, by Bellastella et al. (3), describes a survey tool to facilitate communication from patients to their physicians. The second, by Esfandiari et al. (4), examines physician-patient interactions as perceived by physicians.

\section{Article 1}

\section{Methods}

The aim of the study by Bellastella et al. (3) was to describe a patient survey tool that would help physicians choose the formulation of synthetic thyroxine $\left(T_{4}\right)$ that would be optimal for thyroid hormone replacement in their patients with autoimmune hypothyroidism. Three hundred patients with untreated or not recently treated hypothyroidism secondary to autoimmune thyroiditis were recruited for the study. Inclusion criteria for the study were age $<60$ years, TSH >10 $\mathrm{mlU} / \mathrm{L}$, and "low/normal" serum free $T_{3}$ and free $T_{4}$ levels. Exclusion criteria included a history of any of the following: thyroidectomy, thyroid cancer, central hypothyroidism, diabetes, obesity, current $\mathrm{T}_{4}$ therapy, pregnancy, or intake of a drug that interferes with $\mathrm{L}^{-\mathrm{T}_{4}}$ absorption. Patients were assigned to two groups, each containing 50 men and 100 women. One of the groups, referred to here as E+, was assigned to answer a survey entitled EMPATHY, an acronym 

Important Influences in the Treatment of Hypothyroidism

for "Evaluation of Malabsorption in Patients with Hypothyroidism." The other group, referred to here as $\mathrm{E}-$, did not take the survey.

EMPATHY consisted of seven questions. The first six were designed to uncover a history of drug allergies or intolerance, gastroesophageal reflux, history of Helicobacter pylori infection, inflammatory bowel disease, dietary restrictions, soy intake, or alcohol abuse. The final question presented a check-off panel of foods and food types in various categories, including nickel allergy, lactose intolerance, histamine intolerance, citric acid intolerance, gluten intolerance, and cornstarch allergy.

\section{Results}

At baseline, age and serum thyroid-function tests (free $T_{4}$, free $T_{3}, T S H$ ) were similar in $E+$ and $E$ patients. A drug/food allergy or intolerance was suspected in $30 \mathrm{E}+$ patients based on answers to the questions. Thyroxine treatment was started at a dose of $1.6 \mu \mathrm{g} / \mathrm{kg} /$ day in each patient in the form of tablets, soft gel capsules, or liquid. During the next 6 months, the $\mathrm{T}_{4}$ dose was adjusted to achieve a target TSH of 0.4 to $2.5 \mathrm{mIU} / \mathrm{L}$. During this period, the percentage of tablets and liquid preparations taken by the $\mathrm{E}+$ group was less, but not significantly less, than comparable formulations taken by the E- group. In contrast, approximately 20 percent of patients in the $E+$ group were taking soft-gel $\mathrm{T}_{4}$ preparations, as compared with less than half that number in the $E$ - group $(P=0.002)$.

During the periods 2 months and 6 months after starting $\mathrm{T}_{4}$ therapy, there were significantly more dose adjustments in the $\mathrm{E}$ - group than in the $\mathrm{E}+$ group. Also, whereas all but $4 \%$ of patients in the $E+$ group had reached the target TSH after 6 months, this percentage was $11 \%$ in the $\mathrm{E}-$ group.

\section{Conclusions}

Patient questionnaires that screen for drug and food allergies or intolerances in patients with autoimmune primary hypothyroidism may be useful in selecting an optimal $\mathrm{T}_{4}$ formulation for thyroid hormone replacement.

\section{Article 2}

\section{Methods}

The focus of the study by Esfandiari (4) was to elucidate physicians' perception of the barriers to managing thyroid hormone replacement in patients with hypothyroidism. The authors conducted a survey among randomly chosen physician members of the Endocrine Society (ES), American Academy of Family Practice (AAFP), and American Geriatrics Society (AGS) with regard to their decision-making in treating their patients with hypothyroidism. The goal of the study was to determine whether physician and patient behaviors influence choices for thyroid hormone replacement therapy. The survey consisted of seven sets of questions, each having five response selections ranging from "almost always" to "never,"

or from "very likely" to "not at all likely." Physician responses were dichotomized for analysis. The survey, kindly provided to me by the authors, asked physicians about their practice patterns and interactions with their patients. Respondents were also asked to rate the validity of 14 items, postulated by the authors to be barriers to managing thyroid hormone replacement. Finally, there were several sets of questions about the confidence that physicians had in interventions using decision support tools for managing thyroid hormone replacement.

The survey was mailed to 600 physician addresses; 34 were deemed ineligible for responses that included illness, retirement, or deceased. The response rate 

Important Influences in the Treatment of Hypothyroidism

among the remaining 566 physicians was 63\%. Among these, $36 \%$ were primary care physicians, 32\% endocrinologists, and $32 \%$ geriatricians. The majority of physicians (64\%) had not read guidelines for the treatment of hypothyroidism. Seventy percent of the physicians had prescribed thyroid hormone to 0 to $25 \%$ of their patients; the remaining $30 \%$ prescribed thyroid hormone to 26 to $100 \%$ of their patients.

\section{Results}

Seventy percent of physicians considered it "somewhat likely" to "very likely" that patient nonadherence was a barrier for them in managing thyroid hormone replacement in their patients with hypothyroidism. Interestingly, the next highest percentage of physicians, $41 \%$, rated patient requests for tests and treatments as being a barrier for them in managing thyroid hormone replacement. This perception was positively associated, on multivariate analysis, with endocrinologists and less likely in physicians who had been practicing medicine for more than 20 years. Concern about patient dissatisfaction was noted in $32 \%$ of physicians, and this response was correlated with patient requests for tests and treatments as a barrier to managing thyroid hormone replacement $(P<0.001)$. Providers managing thyroid hormone medications and polypharmacy were almost as numerous, but interestingly, only about a quarter of physicians listed time constraints during clinic visits as a problem.

Approximately $50 \%, 50 \%, 30 \%$, and $20 \%$ of physicians surveyed, respectively, stated that they were very likely or likely to receive various requests from patients including: to adjust their dose of thyroid hormone even when the thyroid-function tests were normal, to use thyroid hormone preparations other than $\mathrm{T}_{4}$, to maintain serum $\mathrm{T}_{4}$ concentrations below normal, and finally, to adjust thyroid hormone doses based on serum free $\mathrm{T}_{3}$ concentrations. As far as what physicians actually did in their practice, 21 percent indicated they sometimes or almost always adjusted the dose of thyroid hormone based on symptoms when thyroid function tests were normal, about 12 percent indicated they would use thyroid hormone preparations other than $\mathrm{T}_{4}, 15$ percent indicated they sometimes or almost always maintained serum TSH concentrations below normal, and about 8 percent indicated they sometimes or almost always adjusted the dose of thyroid hormone based on serum free $T_{3}$ concentrations. The first three of these physician practices were each associated with those physicians who indicated that their patients sometimes, often, or almost always requested these practices $(\mathrm{P}<0.014$ to $\mathrm{P}<0.001)$.

\section{Conclusions}

Physicians perceive patient noncompliance as the most important barrier to managing thyroid hormone replacement in patients with hypothyroidism, but patient requests for tests and treatments outranks other more traditionally perceived barriers. These include multiple providers managing thyroid medication, polypharmacy, clinic-visit time constraints and lack of clinical decision support tools. Perception of patient requests for tests and treatments as a barrier to managing thyroid hormone replacement is associated with physician concern about patient dissatisfaction.

A substantial number of physicians sometimes, often, or always adjust $\mathrm{T}_{4}$ replacement doses based on symptoms even in patients with normal thyroid function tests. Somewhat less than a quarter of physicians sometimes, often or almost always maintain serum TSH below normal. These first two practices may be more prevalent than the physicians using thyroid hormone preparations other than synthetic $\mathrm{T}_{4}$. These three practices, which are contrary to some of the major current guidelines, are more prevalent in physicians who perceive a greater frequency of patient requests for such practices. 

Important Influences in the Treatment of Hypothyroidism

\section{COMMENTARY}

Thyroid hormone is one of the most common prescriptions in the United States, and many patients with hypothyroidism report an impaired quality of life, even when treated. The two articles reviewed here represent different aspects of physician-patient relationships, but both are pertinent to the process shared by physicians and their patients in choosing the formulation and dose of thyroid hormone. The study by Bellastella et al. (3) portrays a concrete, clearly delineated, aspect-the widespread practice of administering surveys to patients for later perusal during office visits. The acronym for the survey was EMPATHY, not to be confused with empathy as an element in the art of medicine (5). Its purpose was to screen for food and drug allergies or intolerances that might be associated with thyroid hormone malabsorption.

The abstract concludes by stating, "Validation of EMPATHY provides endocrinologists with a useful tool in clinical practice, permitting a better personalization of $\mathrm{LT}_{4}$ replacement therapy, a more rapid attainment of the Target TSH levels, and a decreased need for dose adjustments after initiating therapy." In addition to a need for studies with a larger sample size, as indicated by the authors, there are other caveats relating to these conclusions. One is that, because baseline free $T_{4}$ and free $T_{3}$ concentrations ranged from normal to moderately or slightly low, with mild to mild/moderately elevated TSH concentrations, most patients had either subclinical or mild primary hypothyroidism. In the absence of more specific information a reasonable presumption is that the patients' primary physicians used the results of the survey to lead them to suspect increased risks for thyroid hormone malabsorption and then selected a $T_{4}$ formulation that might minimize this risk. This introduces the possibility that the protocol for interpreting and acting on the results of the food and drug intolerance survey was not uniform. For widespread clinical use such surveys need precise and uniform methods to translate survey outcomes into appropriate follow-up. If used as a decision-support tool, this would include computerized data entry and storage, and algorithm generated decision recommendations. The study also reported that all patients were "highly compliant," with no patient recording more than two failures to take the therapy. This conclusion contrasts with the second study by Esfandiari et al. (4) in which physicians viewed noncompliance to be the leading barrier to managing thyroid hormone replacement. Notably, the method to verify medication compliance (3) may have been the only one practical for a 6-month study but is not ideal as it relied on diary records maintained by the patient.

Despite these reservations, a potentially important aspect of the study was that a relatively large percentage of the patients (30\%) that had a history suggestive of drug or food allergy or intolerance. To more completely engage with the patient clinicians might want to incorporate some or all of the questions in the EMPATHY survey into the history they obtain from their hypothyroid patients. At the very least, the results of the survey could be used by physicians to counsel patients regarding optimal dietary habits. As noted by the authors more definitive studies would be needed to diagnose malabsorption.

Whereas the study by Bellastella et al. (3) examines a discrete aspect of physician-patient relationships, that of Esfandiari et al. (4) highlights the myriad complexities of dialog and perception that accompany encounters between patients and providers as they choose thyroid hormone formulations and doses. Before hypothesizing about the implications of physicians' attitudes, it is interesting and pertinent to note some of the tangible findings of the study. One of these is that almost two thirds of the physicians taking the survey had not read guidelines on hypo- 

Important Influences in the Treatment of Hypothyroidism

thyroidism. Moreover, only about $20 \%$ considered unawareness of guidelines to be a barrier to appropriate thyroid hormone management. The reasons for this are not clear, particularly as time constraints during clinic visits were not listed by a large percentage of physicians as a barrier to thyroid hormone management.

In view of the relatively small percentage of physicians who had read the guidelines and the low perception of this as a barrier to managing thyroid hormone replacement, it may not be surprising that many physicians used strategies that did not conform to guidelines for hypothyroidism. Arguably, the most potentially harmful of these is maintaining TSH levels below normal. There is abundant evidence that this increases the risk for adverse cardiovascular outcomes and may also negatively influence longevity.

One of the most novel findings of this study was the high percentage of physicians who had the perception that patients frequently make requests for tests and treatments and that these requests are a barrier to managing thyroid hormone replacement. Interestingly, physicians who perceived this were more likely to be concerned about patient dissat- isfaction. One of the limitations of the study was that the question relating to patient requests for tests and treatments was not parsed by first asking physicians about how often patients make requests for tests and treatments. In those who indicated that these requests were relatively frequent, the next question could be whether they viewed this as a barrier or enhancement to managing thyroid hormone replacement. Hypothetically, those physicians who were most concerned with patient dissatisfaction may tend to perceive patient requests for tests and treatments as a barrier to thyroid hormone management. In contrast, those who perceive such requests as an opportunity to enhance engagement with the patient may be more confident that they are promoting patient satisfaction. In this context it should be noted that patient guidelines, even major and highly adapted guidelines, are dynamic creations that are continually evolving. The most important part of the guidelines is not necessarily the actual recommendations, but rather the studies the recommendations are based on. The best guidelines provide a rich source for the development of physician-patient communication as it relates to what each has to offer. By arriving at more rational and harmonious decisions, well-being for patients and physicians alike is enhanced.

\section{References}

1. Kravitz RL, Bell RA, Azari R, Krupat E, Kelly-Reif S, Thom D 2002 Request fulfillment in office practice: antecedents and relationship to outcomes. Med Care 40:38-51.

2. Kravitz RL, Bell RA, Kelly-Reif S, Krupat E, Thom DH 2003 Direct observation of requests for clinical services in office practice: what do patients want and do they get it? Arch Intern Med 163:1673-1681.

3. Bellastella G, Caputo M, Maiorino MI, Longo M, Scappaticcio L, Giugliano D, Esposito K 2019
EMPATHY: a new tool for identifying the most suitable thyroxine formulation in hypothyroid patients. Thyroid 29:928-933.

4. Esfandiari NH, Reyes-Gastelum D, Hawley ST. Haymart MR, Papaleontiou M 2019 Patient requests for tests and treatments impact physician management of hypothyroidism. Thyroid. Epub 2019 Oct 10.

5. Emerson $\mathrm{CH} 2009$ An element of the art of medicine. Thyroid 19:429-430. 